Cite this: Phys. Chem. Chem. Phys., 2013

\title{
Clarifying the role of sodium in the silica oligomerization reaction
}

15, 1123

Received 17th July 2012, Accepted 16th November 2012

DOI: $10.1039 / \mathrm{c} 2 \mathrm{cp} 42436 \mathrm{c}$

www.rsc.org/pccp

\author{
Anna Pavlova, ${ }^{a}$ Thuat T. Trinh, ${ }^{b}$ Rutger A. van Santen ${ }^{c}$ and Evert Jan Meijer*a
}

\section{Introduction}

Condensation of silicic acid is the elementary reaction step in sol-gel chemistry and zeolite synthesis. Zeolites are crystalline, nanoporous materials with regular channels and cavities that can vary in shape and size for different materials. Their structure can be controlled by the choice of reaction conditions, mainly with adding organic or inorganic salts, commonly referred to as structure directing agents, SDAs. Examples of SDAs are lithium or sodium hydroxides or more complex compounds such as $\mathrm{TPA}^{+}, \mathrm{TMA}^{+}$or $\mathrm{TEA}^{+}$. Due to the variety of different frameworks, zeolites are widely used in the chemical industry for catalysis, absorption and separation. Therefore it is of both scientific and technological interest to understand the process of their formation.

One of the challenges is to explain how silica can oligomerize into different structures depending on the reaction conditions and the nature of the present SDAs. Experimental techniques have been used to investigate the structural changes of the

\footnotetext{
${ }^{a}$ Van't Hoff Institute for Molecular Sciences, University of Amsterdam,

Science Park 904, 1098 XH, Amsterdam, The Netherlands.

E-mail: e.j.meijer@uva.nl; Tel: +31 205256448

${ }^{b}$ Department of Chemistry, Norwegian University of Science and Technology, 7491, Trondheim, Norway

${ }^{c}$ Chemical Engineering and Chemistry Department, Eindhoven University of Technology, P.O. Box 513, Eindhoven, The Netherlands
}

species during a zeolite formation process. ${ }^{1-9}$ It is known that this process consists of several parallel reactions and that the final shape of the framework is determined at the early stages of the oligomerization. In the initial stage, small oligomers such as dimers, trimers, tetramers, 3-rings, 4-rings, or others are formed. ${ }^{3}$ The distribution of the different structures depends strongly on the reaction conditions such as $\mathrm{pH}$, temperature, silica concentrations and type of SDAs. This distribution of small oligomers is correlated with the final structure..$^{5-9}$

To arrive at a better understanding of silica oligomerization, several theoretical and experimental studies have addressed the effect of the environment on the first stages of silica condensation. ${ }^{1,2,4-11}$ The evolution of different silica species under different reaction conditions has been studied by Burkett and Davis ${ }^{6,7}$ and Pelster et al. ${ }^{11}$ It has also been shown that the presence of cations can significantly affect the formation rate of the smaller oligomers. ${ }^{2,10}$ However, due to the formation of several silica oligomers simultaneously it is difficult to study the formation of a single silica oligomer accurately with experimental techniques. In addition, it is difficult to disentangle the role of the individual reaction condition variables.

Theoretical methods do not have these limitations. Various theoretical studies have investigated the mechanism of $\mathrm{Si}-\mathrm{O}-\mathrm{Si}$ linking of silicic acid, concluding that an ionic two-step mechanism, shown in Scheme 1, is most favorable. ${ }^{12,13}$ In this mechanism, 

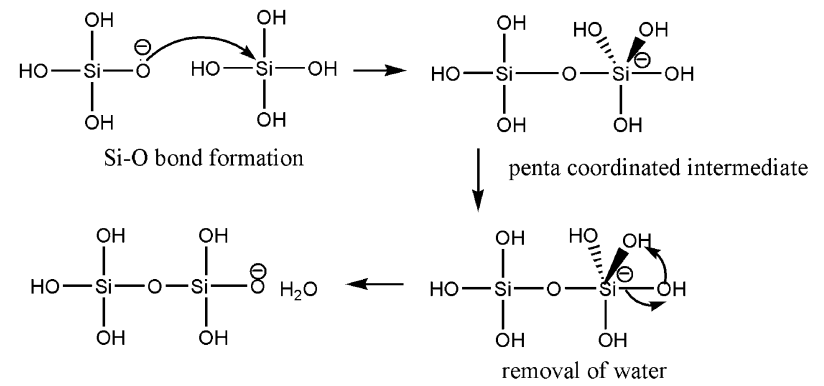

Scheme 1 The mechanism of the silica condensation reaction.

a negatively charged silica oxygen attacks the silicon of another monomer as the first step, yielding a penta-coordinated intermediate. Then water is removed in the second step. Further studies showed that energy barriers of formation and stability can differ significantly for different silica oligomers, mainly due to the importance of intramolecular hydrogen bonds. Tossell ${ }^{14}$ and Pereira et al. ${ }^{15,16}$ have studied the effect of solvent on the reaction using continuum models, concluding that a continuum solvent model significantly changes the reaction barriers. Catlow et al. and Lewis et al. have studied the role of organic cations and shown that they can play an important role by stabilizing the growing silica oligomers and by directing their growth. ${ }^{17,18}$ Mora-Fonz et al. showed that the presence of a sodium ion can affect $\mathrm{p} K_{\mathrm{a}}$ values of silica oligomers. ${ }^{19}$

Trinh et al. ${ }^{20,21}$ did ab initio molecular dynamics simulations for this reaction in explicit water solution. The barriers obtained with the explicit solvent model differ significantly from those obtained from studies in gas phase complemented by a cosmo continuum model. ${ }^{12-16}$ In addition, in some cases a different reaction mechanism, for the second step, was observed. In the new mechanism a water molecule would transfer the proton to the leaving $\mathrm{OH}$ group instead of a $\mathrm{Si}-\mathrm{OH}$ group.

They also studied the effect of lithium and ammonium ions on the formation of smaller silicic acid chains using the same method. ${ }^{22}$ It was shown that cations increase the total reaction barrier and that the $\mathrm{Si}-\mathrm{O}$ bond formation step is more affected than the water removal step. For the silica dimer formation, the cations were observed to directly coordinate to the reacting $\mathrm{Si}-\mathrm{O}$ group during the first reaction step, explaining the increased barriers in these cases. These studies show that including an explicit aqueous environment is important for accurate theoretical studies of the silica condensation reaction.

Sodium is a commonly used additive in zeolite synthesis. It has been shown to decrease the reaction rate of silica condensation at early stages, ${ }^{2}$ yet increase crystallization and growth of zeolites at later stages. ${ }^{7}$ Nevertheless, its effect on silica condensation has not been studied theoretically in explicit solution. Based on the previous studies ${ }^{2,7,10,19}$ it was reasonable to assume that sodium does have an effect on the early stages of silica condensation reaction. In the present paper we address the role of sodium, and report an ab initio molecular dynamics study of silica condensation reaction in aqueous solution in the presence of a sodium ion.

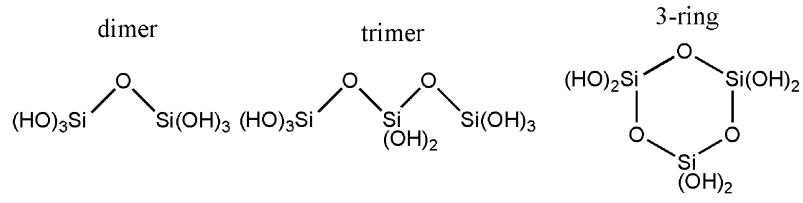

Scheme 2 The silica oligomers, formation of which was studied.

\section{Methods}

We have studied dimerization, trimerization and 3-ring closure reactions (Scheme 2) in explicit water solution in the presence of a sodium ion. The solvent was represented by 64 water molecules in a periodic cubic box, which provided two solvation shells. We did not include the hydroxide ion, in order to obtain insight into the explicit effects of the sodium ion, and to be able to make a direct comparison with previous computational studies that also excluded the hydroxide ion.

The electronic structure was calculated using the KohnSham formulation $^{23}$ of Density Functional Theory, ${ }^{24}$ DFT. The BLYP density functional ${ }^{25,26}$ was used. It provides a sufficiently accurate description of water ${ }^{27}$ and water-silica interactions. ${ }^{19}$ The Kohn-Sham orbitals were expanded in a plane wave basis set with that included plane waves up to an energy of $70 \mathrm{Ry}$. Norm-conserving Troullier Martin pseudopotentials ${ }^{28,29}$ were used to represent the non-valence electrons. The pseudopotential cutoff radii for $\mathrm{Si}, \mathrm{Na}, \mathrm{O}$ and $\mathrm{H}$ were 1.9, 0.98, 1.05 and 0.50 a.u., respectively, for the $\mathrm{s}$ term. We used the same cutoff for the $\mathrm{p}$ term as for the $\mathrm{s}$ term for $\mathrm{O}$ and $\mathrm{Na}$, while for $\mathrm{Si}$ we used a cutoff of 2.1 a.u. for the $\mathrm{p}$ term. The CPMD program package was used for all calculations. ${ }^{30}$

The reaction was studied with Car-Parrinello molecular dynamics. ${ }^{31}$ It is an $a b$ initio molecular dynamics technique where the plane-wave coefficients are given a fictitious mass and are propagated dynamically such that the electronic state remains near the ground state. We used a fictitious mass of 600 a.u., which is sufficiently small for a good description of water. $^{32}$ Deuterium was used instead of hydrogen in order to use a larger time step. These settings allowed for a time step of 0.121 fs. The simulations were done in an NVT ensemble. The volume of the simulation box corresponded to a density of $1 \mathrm{~g} \mathrm{~cm}^{-3}$ and the temperature was $350 \mathrm{~K}$. Constant temperature was imposed using a Nosé-Hoover thermostat ${ }^{33}$ with a frequency of $500 \mathrm{~cm}^{-1}$.

The time scales at which the silica oligomerization reactions occur are not accessible with today's ab initio dynamics methods. Therefore, we used the constrained dynamics method to force

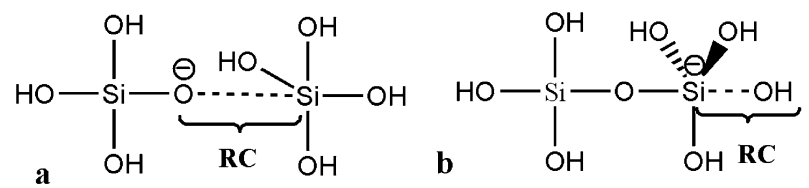

Scheme 3 The reaction coordinates, $\mathrm{RC}$, chosen to model silica condensation reaction. (a) RC for the Si-O bond formation step. (b) RC for the water removal step. 
the reaction to occur. ${ }^{34}$ The Si-O distance of the attacking oxygen was chosen as the reaction coordinate for the first step of the reaction, while for the second step, the reaction coordinate was the $\mathrm{Si}-\mathrm{O}$ distance of the leaving $\mathrm{OH}^{-}$group, see Scheme $3 \mathrm{a}$ and $\mathrm{b}$.

We performed constrained molecular dynamics simulations with chosen values of the reaction coordinate. For each value a 2 ps equilibration run was followed by a 10 ps production run. The average constrained force was calculated for the production runs. The free energy profiles of the reactions were obtained with thermodynamic integration, as in equation (1), where $F$ is the constrained force and $r$ is the reaction coordinate.

$$
\Delta G=-\int_{r_{1}}^{r_{2}}\langle F(r)\rangle \mathrm{d} r
$$

\section{Results and discussion}

In order to validate that our DFT setup properly describes the solvation of sodium we calculated the hydration energies of sodium for one to four water molecules. The results are in agreement with experimental energies ${ }^{35}$ and previous DFT calculations, ${ }^{36}$ see Table 1.

We also have investigated the solvation of sodium by calculating the $\mathrm{Na}-\mathrm{O}$ radial distribution function and coordination number for sodium in the case of the silica dimer, see Fig. 1. The graph was obtained from a 30 ps long unconstrained ab initio MD simulation. The first peak is well defined, it shows that there is a clear structure for the first solvation shell of the

Table 1 Calculated hydration energies for $\mathrm{Na}^{+}\left(\mathrm{H}_{2} \mathrm{O}\right)_{n}$ clusters in $\mathrm{kcal} \mathrm{mol}^{-1}$. An isolated box of $15 \AA$ was used for all of the calculations. Experimental hydration energies $^{35}$ and previous DFT calculations ${ }^{36}$ are added for comparison

\begin{tabular}{llll}
\hline No. & Our results & Previous DFT results & Experimental results \\
\hline 1 & 23.9 & 23.4 & 23.4 \\
2 & 20.8 & 20.1 & 19.2 \\
3 & 16.6 & 17.1 & 15.2 \\
4 & 13.2 & 13.3 & 13.2
\end{tabular}

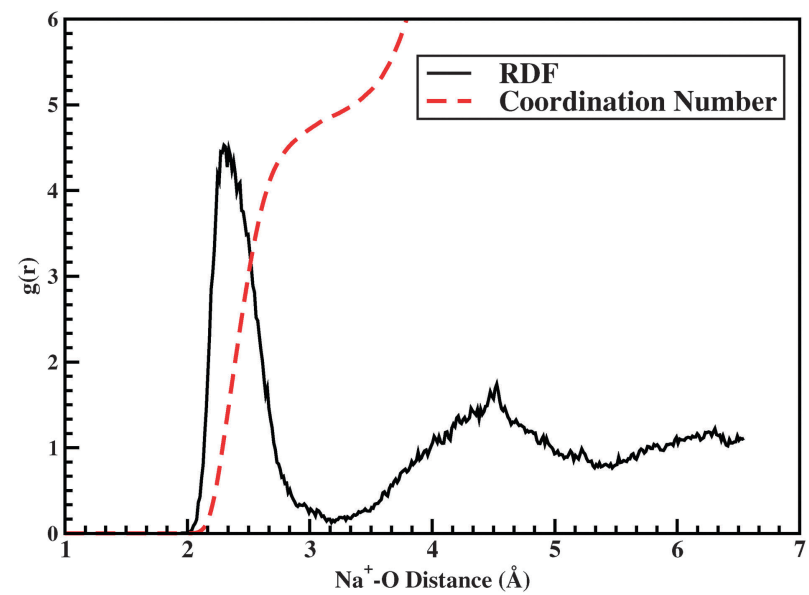

Fig. 1 Radial distribution function for the $\mathrm{Na}-\mathrm{O}$ pair and the coordination number obtained from it. sodium ion. The first peak is located between 2.1 and $3.2 \AA$ and the second peak between 3.2 and $5.4 \AA$. The coordination number in the first shell is 4.8 which is consistent with previous theoretical and experimental studies. ${ }^{37-39}$

For all of the three reactions and for both reaction steps, the constrained force was sampled for values between 3.8 and $1.85 \AA$, with the intervals of $0.1-0.2 \AA$. The end points of the force profiles were chosen such that the constrained force was sufficiently close to 0 . The force profiles for all of the three reactions, see Fig. 2, look similar.

The free energy profiles obtained from the integration of the forces are shown in Fig. 3. The energies for the water removal step were obtained by integrating the constrained force and adding the final energy of the $\mathrm{Si}-\mathrm{O}$ bond formation step. The profiles for dimer and trimer are almost indistinguishable, while 3-ring formation has a lower barrier for Si-O linking and a higher barrier for the water removal step. Table 2 shows the exact values for each step and each reaction.

The total free energy of the studied reactions is unfavorable and this is consistent with studies in pure water by Trinh et al. ${ }^{20}$ They analyzed the internal energy changes of the system and found that the reaction was exothermic, as in the experimental studies. Therefore, the reaction free energies were unfavorable due to the significant decrease of the entropy. One water molecule is produced in the silica oligomerization reaction. According to experimental ${ }^{40}$ and theoretical studies, ${ }^{41} T \Delta S$ of water dimer formation is around $30 \mathrm{~kJ} \mathrm{~mol}^{-1}$ at $373 \mathrm{~K}$. Therefore, the rearrangement of the hydrogen bond network of water due to the formation of one more water molecule could be significant enough to make an exothermic reaction unfavorable.

The accuracy of DFT for reaction barriers is estimated to be 5-10 $\mathrm{kJ} \mathrm{mol}^{-1}$. The differences in the total reaction barriers for the three studied reactions are within these errors. However, the 3-ring formation has a lower barrier for $\mathrm{Si}-\mathrm{O}$ bond formation and a higher barrier for the water removal step. Thus the total reaction barrier remains close to the dimer and trimer. Fig. 4 and Table 2 compare the total reaction barriers in the presence of $\mathrm{Na}^{+}$to previously calculated cases without any cation ${ }^{20}$ and in the presence of $\mathrm{Li}^{+} .^{22}$ The difference between sodium and lithium is much larger than the error margin, while the difference between pure water and sodium is larger than the error margin only for dimer and trimer formations.

We studied the trajectories in order to investigate if the observed differences in the reaction barriers are due to the direct coordination of sodium to the reactants or because of its effect on the hydrogen bond network of water. Table 2 shows that the differences for the $\mathrm{Si}-\mathrm{O}$ bond formation step are larger than the differences for the water removal step. Fig. 5 shows representative snapshots for observed $\mathrm{Na}^{+}$coordinations for the $\mathrm{Si}-\mathrm{O}$ bond formation step. Direct coordination to the negatively charged oxygen that is forming the $\mathrm{Si}-\mathrm{O}$ bond, see Fig. 5a, is rare for all of the three reactions. This type of coordination is common when $\mathrm{Li}^{+}$is used ${ }^{22}$ instead of $\mathrm{Na}^{+}$ and could explain the difference in the energy barriers for the 


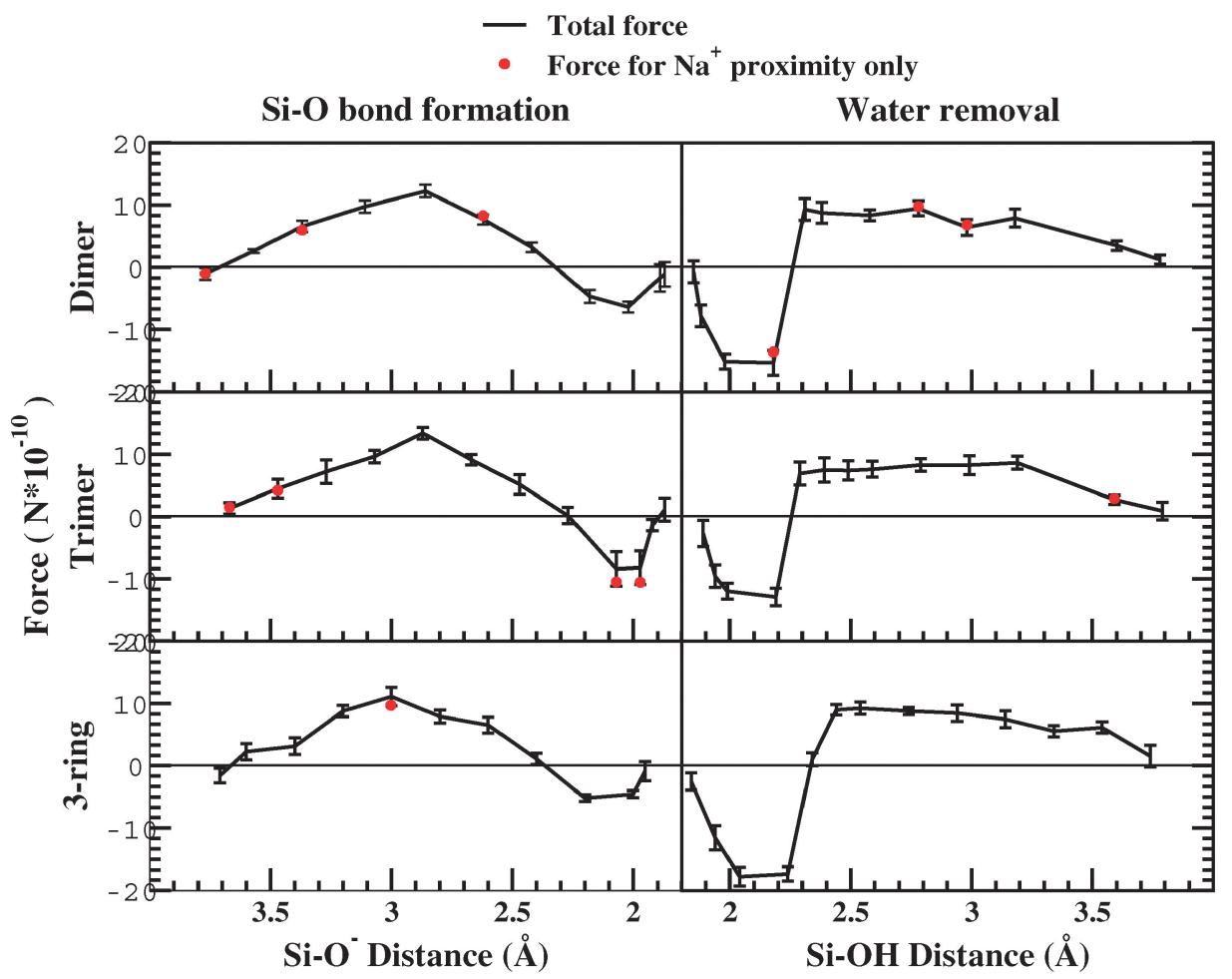

Fig. 2 Calculated constrained force profiles along the reaction coordinates for the three reactions. The statistical errors for the total forces are shown as in the $95 \%$ confidence interval. The average constrained force for the configurations where sodium is within $3.2 \AA$ of a Si-OH group are shown as circles. These averages where only calculated for samples where sodium is close by for at least 3 ps and far away for at least 3 ps.

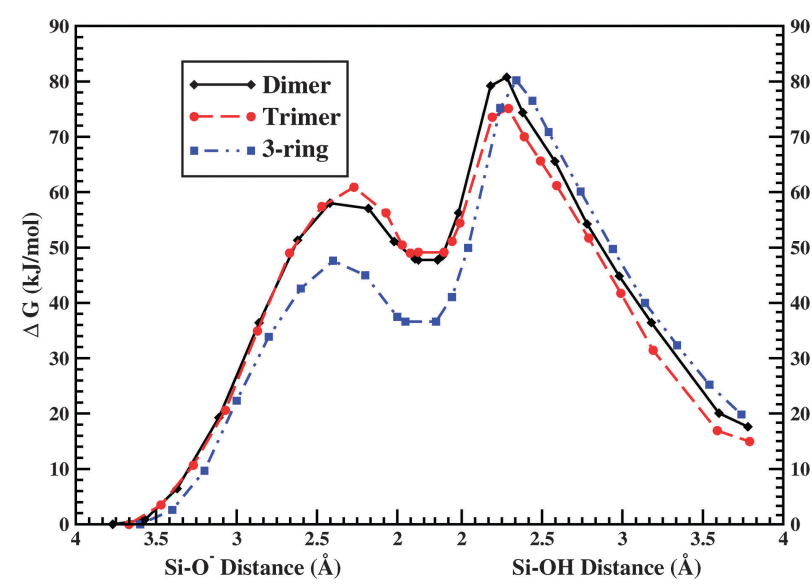

Fig. 3 Calculated free energy profiles as functions of the reaction coordinate.

two ions. Coordination to non-reacting $\mathrm{Si}-\mathrm{OH}$ groups, see Fig. $5 \mathrm{~b}$, is more common than to the negatively charged oxygen. However, in the most common case, the sodium ion is either far away or in the second solvation shell. Fig. 5c shows a snapshot where sodium is in the second solvation shell of the reactive oxygen. Note, when sodium is close to the negatively charged oxygen, this oxygen has only one hydrogen bond. When sodium is far away, this oxygen can have up to three hydrogen bonds. This could explain why this coordination is rarely observed, two hydrogen bonds could be more favorable
Table 2 Calculated free energies for dimer, trimer and 3-ring formation in the presence of sodium. The energies without any cation ${ }^{20,21}$ and in the presence of $\mathrm{Li}^{+}$(ref. 22) are added for comparison. All energies are in $\mathrm{kJ} \mathrm{mol}^{-1}$

\begin{tabular}{lllr}
\hline Reaction & No cations present & $\mathrm{Na}^{+}$ & $\mathrm{Li}^{+}$ \\
\hline Dimerization & & & \\
First step & 44 & 58 & 70 \\
Second step & 42 & 33 & 39 \\
Total & 61 & 81 & 98 \\
Trimerization & & & \\
First step & 43 & 61 & 78 \\
Second step & 26 & 26 & 46 \\
Total & 53 & 75 & 108 \\
3-Ring formation & & & \\
First step & 35 & 48 & 83 \\
Second step & 48 & 44 & 44 \\
Total & 72 & 80 & 111 \\
& & &
\end{tabular}

than coordination to the sodium ion. Table 3 summarizes how often the sodium ion is close to silica for each of the three reactions.

During the water removal step, the leaving $\mathrm{OH}^{-}$group receives a proton. For all of the three reactions studied here this proton comes from a water molecule and a hydroxide ion is formed for a short time. At a later stage a $\mathrm{Si}-\mathrm{OH}$ group is deprotonated, neutralizing the hydroxide. Both of these proton transfers go back and forward a few times before they are completed, suggesting that they are reversible. Fig. 6 shows selected snapshots around the transitions state for the three reactions. For dimer formation, deprotonation of a 


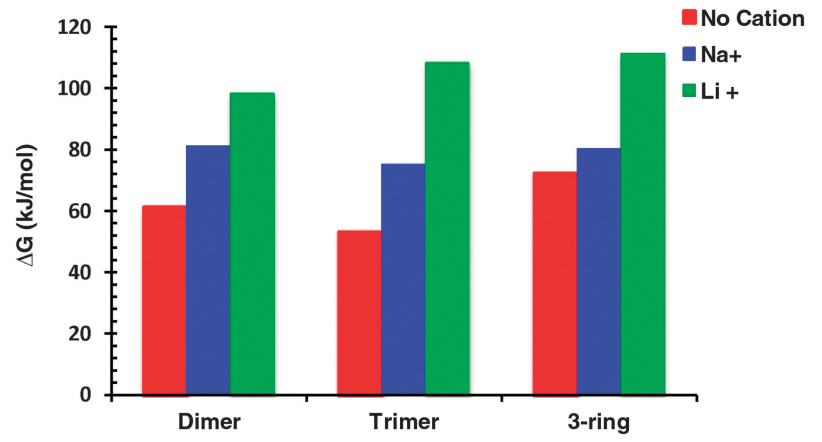

Fig. 4 Comparison of the calculated free energy barriers with sodium to those without any cation ${ }^{20,21}$ and with lithium. ${ }^{22}$

$\mathrm{Si}-\mathrm{OH}$ group occurs within $3 \mathrm{ps}$ of the proton transfer to the leaving $\mathrm{OH}^{-}$group, while for the trimer and 3-ring formation it occurs within 5 ps. During the deprotonation of a $\mathrm{Si}-\mathrm{OH}$ group, the proton is immediately shuttled to the previously formed hydroxide group through a hydrogen bond network, neutralizing it. In the case of dimer and trimer, one of the $\mathrm{Si}-\mathrm{OH}$ groups on the reacting silica atom is pointing towards the leaving group, resulting in a direct proton transfer to the hydroxide, as shown in Fig. 6a and b. In the case of 3-ring formation, all of the $\mathrm{Si}-\mathrm{OH}$ groups of the reacting silicon atom are pointing away from the leaving $\mathrm{OH}^{-}$group. Therefore, a $\mathrm{Si}-\mathrm{OH}$ group from a non-reacting silicon atom is deprotonated instead, resulting in a spatially more extended proton transfer network. Note that the sodium is far away from the reaction center in all three cases.

If direct coordination of the sodium ion to the $\mathrm{Si}-\mathrm{O}^{-} / \mathrm{Si}-\mathrm{OH}$ groups is the dominant reason for the increase of the reaction barriers, it should be reflected in the value of the constrained force for configurations with direct coordination. To investigate if this was the case, we calculated the averages of the constrained force for snapshots where $\mathrm{Na}^{+}$was within $3.2 \AA$ of a $\mathrm{Si}-\mathrm{O}$ group. In order to get good statistics we only considered points along the reaction coordinate where sodium
Table 3 Percent of snapshots during the whole reaction, for which $\mathrm{Na}^{+}$is within $3.2 \AA$ of any Si-O group

\begin{tabular}{llll}
\hline Reaction & Dimer & Trimer & 3-Ring \\
\hline Proximity in \% & 21 & 20 & 5 \\
\hline
\end{tabular}

spent at least 3 ps of time coordinated and at least 3 ps far away. The average of the constrained force when sodium is close to a $\mathrm{Si}-\mathrm{O}$ group was compared to the total average for these points. The differences in the forces, shown in Fig. 2, are smaller than the statistical errors. This strongly suggests that this type of coordination does not have a significant effect on the constrained force and consequently on the reaction barrier.

Although sodium does not have an increasing effect on the reaction barriers due to direct coordination, it could still have an effect while being sufficiently close to affect the hydrogen bond network around the reactants. In order to investigate if this is the case, we analyzed the hydrogen bonding of the reacting oxygen in the $\mathrm{Si}-\mathrm{O}$ bond formation step of the reaction. The top part of Fig. 7 shows the number of hydrogen bonds for this oxygen totally, only with silica and only with water during the reaction. We only counted the bonds that are shorter than $2.2 \AA$, which is slightly higher than the average hydrogen bond length in water which is $1.97 \AA$. The bottom part of Fig. 7 shows fraction of frames where sodium is either in the first or the second shell of the reacting oxygen during the corresponding run. Based on the $\mathrm{Na}-\mathrm{O}$ RDF in Fig. 1, we counted the configurations in which the distance between sodium and the reactive oxygen is less than $5.4 \AA$. When the reaction coordinate is between 3.7 and $3.1 \AA$, the total number of hydrogen bonds decreases with decreasing reaction coordinate for dimer and trimer formation and fluctuates for 3-ring formation. These changes do not appear to correlate with sodium proximity in this region. However, when the reaction coordinate is between 3.1 and $2.2 \AA$, just before the transition state, there is a correlation between the decrease in the total number of hydrogen bonds and sodium proximity to the

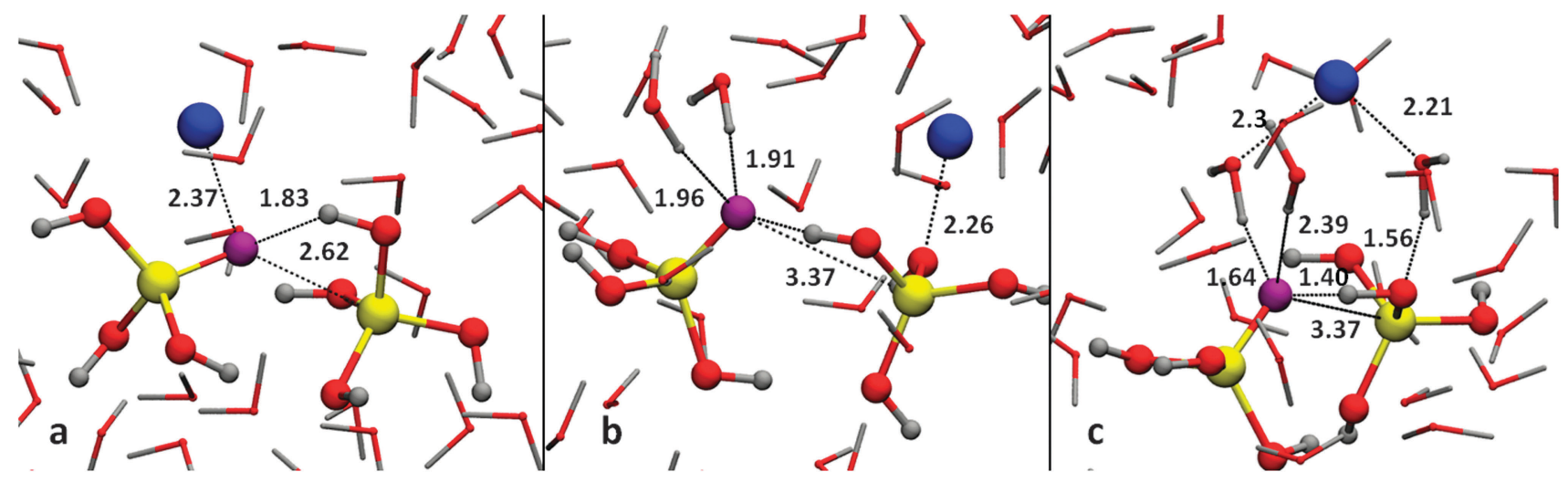

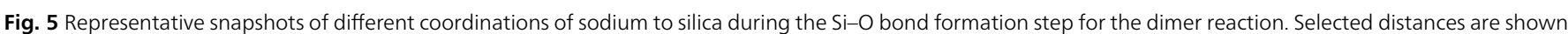

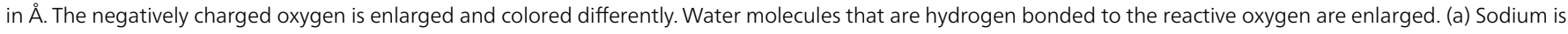

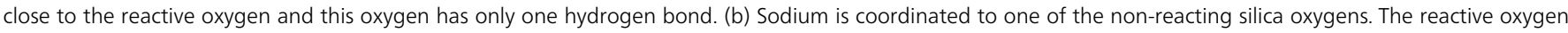
has three hydrogen bonds. (c) The sodium ion is in the second solvation shell of the reactive oxygen. The reactive oxygen has three hydrogen bonds. One of the water molecules participating in the hydrogen bonding is also coordinated to the sodium ion. 


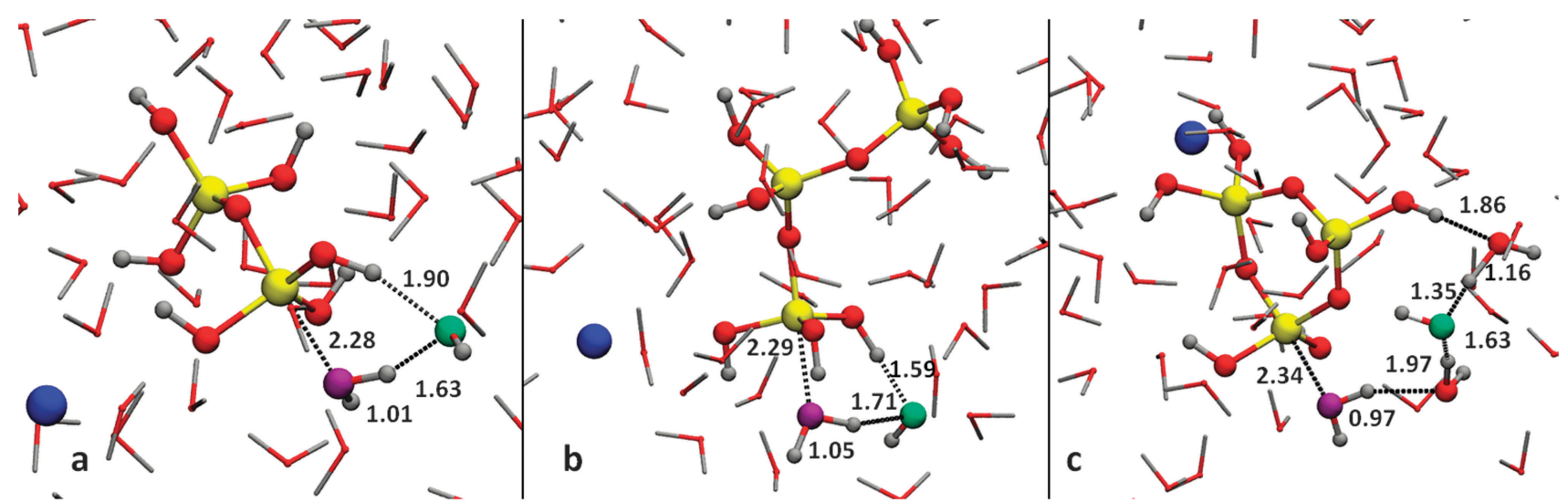

Fig. 6 Representative snapshots of the water removal step just after the proton transfer. The hydrogen bonds important for the proton transfers are shown and selected distances are displayed in $\AA$. For clarity the oxygens of the leaving $\mathrm{OH}^{-}$and the hydroxide group are colored purple and green respectively. (a) Dimer formation. (b) Trimer formation. (c) 3-Ring formation, the hydrogen bond network that deprotonates the silica structure is larger here.

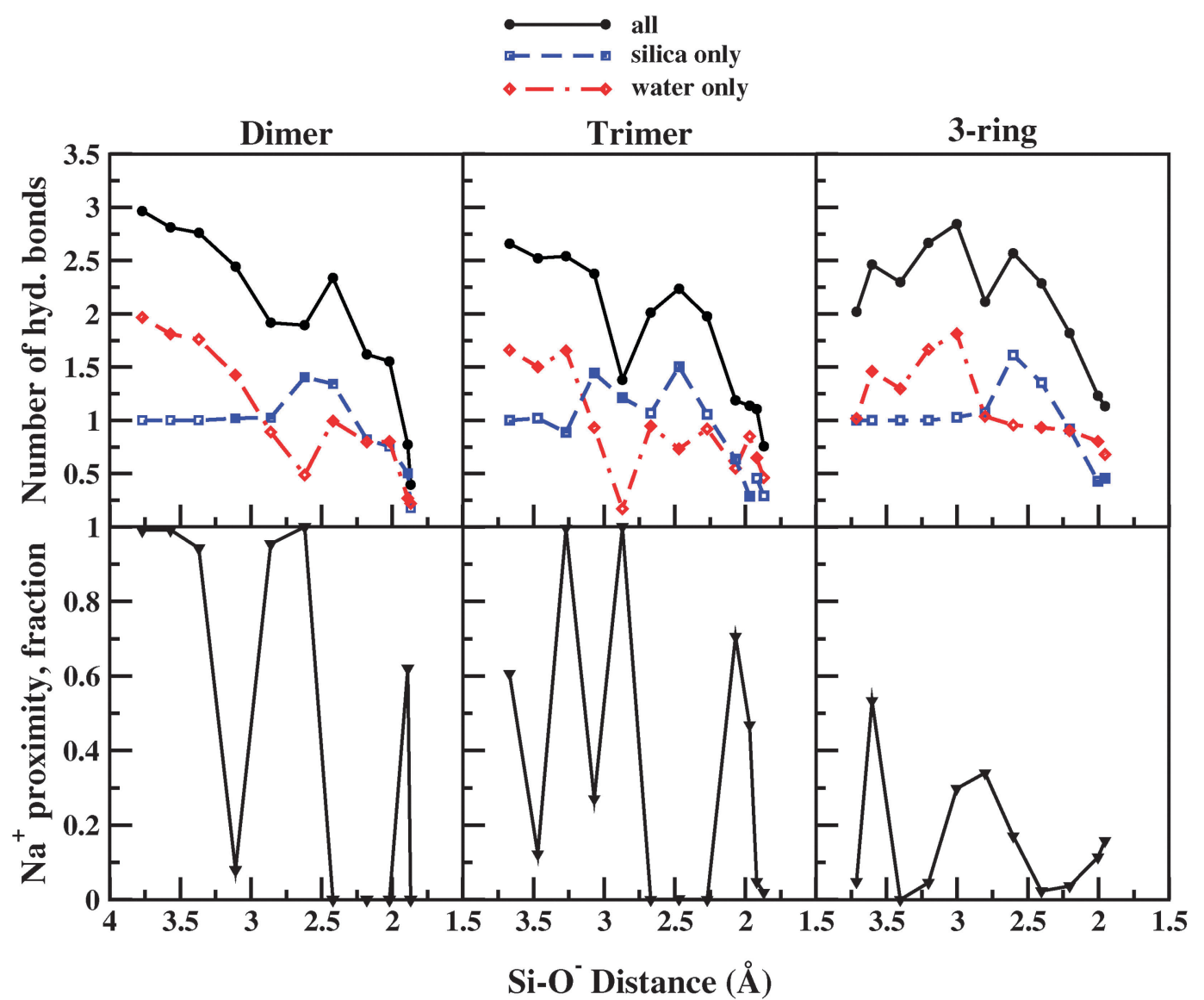

Fig. 7 Top figures show the average number of hydrogen bonds for the reacting oxygen during the first reaction step of dimer, trimer and 3-ring formation. They also show the average number of hydrogen bonds with only silica and with only water molecules for this oxygen. Only bonds shorter than $2.2 \AA$ were counted. Bottom figures show a frequency of sodium proximity to the reacting oxygen, in fraction. The $\mathrm{Na}-\mathrm{O}$ distance shorter than $5.4 \AA$ was counted as proximity.

reacting oxygen. Fig. 7 also shows that it is hydrogen bonding with the water molecules and not the other silica that decreases when sodium is close. This decrease in hydrogen bonding with water molecules could result in a higher reaction barrier. Furthermore, in the case of 3-ring formation, sodium proximity to the reactive oxygen is less frequent than for dimer and trimer formation. The smaller effect on the 3-ring formation barrier than on dimer and trimer formation barriers when sodium is present could be explained by less frequent sodium proximity to the reactive oxygen. 


\section{Conclusions}

We have studied formation of silica dimer, trimer and ring closure in explicit water solution with sodium counter ions using ab initio molecular dynamics. Our results show that sodium increases the reaction barriers of smaller oligomers compared to the case with no counter ion for all of the three reactions $\mathrm{s}^{20,21}$ and that these effects are smaller than when a lithium ion is used. ${ }^{22}$ Just as in a previous theoretical study, ${ }^{22}$ the $\mathrm{Si}-\mathrm{O}$ bond formation step is significantly more affected by the presence of a cation than the water removal step. The increase of reaction barriers for smaller oligomers when sodium is present is consistent with an experimental study under basic conditions. ${ }^{10}$ While a study by Harrison and Loton ${ }^{2}$ reports that dimer and trimer formation is slightly slower with sodium than with lithium, these experiments are done at neutral $\mathrm{pH}$. This suggests that behavior of the cations in silica oligomerization can be sensitive to $\mathrm{pH}$ variations. Furthermore, these experimental conditions also include catechol, which was not included in this theoretical study. Since catechol has two $\mathrm{OH}$ groups, it could interact with cations and change their behavior during the reaction. The three obtained reaction barriers are very close, suggesting that all of the three reactions are equally favored in the presence of sodium.

We also investigated possible reasons for increased reaction barriers. We found that direct coordination to the negatively charged oxygen, observed for other ions ${ }^{22}$ was rare for sodium. A possible reason for this is that coordination to sodium leads to breaking of two hydrogen bonds for the negatively charged oxygen, making the coordination unfavorable. Instead sodium was often coordinated to non-reacting $\mathrm{Si}-\mathrm{OH}$ groups or present in the second solvation shell. We found that coordination to the $\mathrm{Si}-\mathrm{OH}$ groups did not affect the constrained force and therefore could not be the reason for the increased barriers. However, sodium was often present in the second solvation shell of the reactive oxygen during the $\mathrm{Si}-\mathrm{O}$ bond formation step of the dimer and trimer reaction. This was not the case for the ring closure reaction, for which the increase in the reaction barrier was less dramatic. Hydrogen bonding of the reactive oxygen during the $\mathrm{Si}-\mathrm{O}$ bond formation was analyzed. We found correlation between sodium presence in either the first or the second solvation shell of the oxygen and a decrease in its hydrogen bonding just before the transition state. Therefore, sodium could influence the reaction barriers by inducing some rearrangement of the hydrogen-bond network of water solution around the reactants. To summarize, our result shows that sodium can play an important role in silica oligomerization reaction by increasing the reaction barriers, although it does not directly coordinate to the reaction center. Further studies are needed on the role of $\mathrm{pH}$ and on the interaction of sodium with other present SDAs. In addition, improved insight into the dynamics of the role of sodium and other SDAs could be gathered from modeling reactive pathways using transition path sampling. To extend the scope in the context of zeolite synthesis the present approach can be extended to include metal cations, such as aluminum or boron, that are commonly present in zeolites.

\section{References}

1 C. E. A. Kirschhock, R. Ravishankar, F. Verspeurt, P. J. Grobet, P. A. Jacobs and J. A. Martens, J. Phys. Chem. B, 1999, 103, 4965-4971.

2 C. C. Harrison and N. Loton, J. Chem. Soc., Faraday Trans., 1995, 91, 4287-4297.

3 C. S. Cundy and P. A. Cox, Chem. Rev., 2003, 103, 663-702.

4 S. Zones, Microporous Mater., 1994, 2, 281-287.

5 P. Bussian, F. Sobott, B. Brutschy, W. Schrader and F. Schüth, Angew. Chem., Int. Ed., 2000, 39, 3901-3905.

6 S. L. Burkett and M. E. Davis, Chem. Mater., 1995, 7, 920-928.

7 S. L. Burkett and M. E. Davis, Chem. Mater., 1995, 7, 1453-1463.

8 F. Gaboriaud, A. Nonat, D. Chaumont, A. Craievich and B. Hanquet, J. Phys. Chem. B, 1999, 103, 2091-2099.

9 C.-F. Wang, J.-S. Li, L.-J. Wang and X.-Y. Sun, J. Hazard. Mater., 2008, 155, 58-64.

10 A. V. McCormick, A. T. Bell and C. J. Radke, J. Phys. Chem., 1989, 93, 1737-1741.

11 S. A. Pelster, W. Schrader and F. Schüth, J. Am. Chem. Soc., 2006, 128, 4310-4317.

12 T. T. Trinh, A. P. J. Jansen and R. A. van Santen, J. Phys. Chem. B, 2006, 110, 23099-23106.

13 J. C. G. Pereira, C. R. A. Catlow, J. C. G. Pereira and G. D. Price, Chem. Commun., 1998, 1387-1388.

14 J. Tossell, Geochim. Cosmochim. Acta, 2005, 69, 283-291.

15 J. C. G. Pereira, C. R. A. Catlow and G. D. Price, J. Phys. Chem. A, 1999, 103, 3252-3267.

16 J. C. G. Pereira, C. R. A. Catlow and G. D. Price, J. Phys. Chem. A, 1999, 103, 3268-3284.

17 D. W. Lewis, C. M. Freeman and C. R. A. Catlow, J. Phys. Chem., 1995, 99, 11194-11202.

18 C. R. A. Catlow, D. S. Coombes, D. W. Lewis and J. C. G. Pereira, Chem. Mater., 1998, 10, 3249-3265.

19 M. J. Mora-Fonz, C. R. A. Catlow and D. W. Lewis, J. Phys. Chem. C, 2007, 111, 18155-18158.

20 T. T. Trinh, A. P. J. Jansen, R. A. van Santen and E. Jan Meijer, Phys. Chem. Chem. Phys., 2009, 11, 5092.

21 T. T. Trinh, A. P. J. Jansen, R. A. van Santen and E. J. Meijer, J. Phys. Chem. C, 2009, 113, 2647-2652.

22 T. T. Trinh, A. P. J. Jansen, R. A. van Santen, J. VandeVondele and E. J. Meijer, ChemPhysChem, 2009, 10, 1775-1782.

23 W. Kohn and L. J. Sham, Phys. Rev., 1965, 140, A1133-A1138.

24 P. Hohenberg and W. Kohn, Phys. Rev., 1964, 136, B864-B871.

25 A. D. Becke, Phys. Rev. A: At., Mol., Opt. Phys., 1988, 38, 3098-3100.

26 C. Lee, W. Yang and R. G. Parr, Phys. Rev. B: Condens. Matter Mater. Phys., 1988, 37, 785-789.

27 M. Sprik, J. Hutter and M. Parrinello, J. Chem. Phys., 1996, 105, 1142-1152.

28 N. Troullier and J. L. Martins, Phys. Rev. B: Condens. Matter Mater. Phys., 1991, 43, 8861.

29 N. Troullier and J. L. Martins, Phys. Rev. B: Condens. Matter Mater. Phys., 1991, 43, 1993.

30 CPMD, http://www.cpmd.org/, Copyright IBM Corp. 1990-2008, Copyright MPI für Festkörperforschung Stuttgart 1997-2001.

31 R. Car and M. Parrinello, Phys. Rev. Lett., 1985, 55, 2471-2474.

32 E. Schwegler, J. C. Grossman, F. Gygi and G. Galli, J. Chem. Phys., 2004, 121, 5400-5409.

33 S. Nosé, J. Chem. Phys., 1984, 81, 511-519.

34 E. Carter, G. Ciccotti, J. T. Hynes and R. Kapral, Chem. Phys. Lett., 1989, 156, 472-477.

35 I. V. Hertel, C. Hüglin, C. Nitsch and C. P. Schulz, Phys. Rev. Lett., 1991, 67, 1767-1770.

36 L. M. Ramaniah, M. Bernasconi and M. Parrinello, J. Chem. Phys., 1998, 109, 6839-6843.

37 S. B. Rempe and L. R. Pratt, Fluid Phase Equilib., 2001, 183-184, 121-132.

38 S. Varma and S. B. Rempe, Biophys. Chem., 2006, 124, 192-199.

39 N. Galamba and B. J. Costa Cabral, J. Phys. Chem. B, 2009, 113, 16151-16158.

40 L. A. Curtiss, D. J. Frurip and M. Blander, J. Chem. Phys., 1979, 71, 2703-2711.

41 O. Isayev, L. Gorb and J. Leszczynski, J. Comput. Chem., 2007, 28, 1598-1609. 